\title{
Prosodic strengthening and featural enhancement: Evidence from acoustic and articulatory realizations of /a,i/ in English
}

\author{
Taehong Cho ${ }^{\text {a) }}$ \\ Division of English Language and Literature, Hanyang University, 17 Haengdang-dong, Seongdong-gu, \\ Seoul, 133-791, Korea, and Max Planck Institute for Psycholinguistics, Nijmegen, The Netherlands
}

(Received 20 September 2002; revised 5 January 2005; accepted 5 January 2005)

\begin{abstract}
In this study the effects of accent and prosodic boundaries on the production of English vowels $(/ \mathrm{a}, \mathrm{i} /)$, by concurrently examining acoustic vowel formants and articulatory maxima of the tongue, jaw, and lips obtained with EMA (Electromagnetic Articulography) are investigated. The results demonstrate that prosodic strengthening (due to accent and/or prosodic boundaries) has differential effects depending on the source of prominence (in accented syllables versus at edges of prosodic domains; domain initially versus domain finally). The results are interpreted in terms of how the prosodic strengthening is related to phonetic realization of vowel features. For example, when accented, /i/ was fronter in both acoustic and articulatory vowel spaces (enhancing [-back]), accompanied by an increase in both lip and jaw openings (enhancing sonority). By contrast, at edges of prosodic domains (especially domain-finally), /i/ was not necessarily fronter, but higher (enhancing [+high]), accompanied by an increase only in the lip (not jaw) opening. This suggests that the two aspects of prosodic structure (accent versus boundary) are differentiated by distinct phonetic patterns. Further, it implies that prosodic strengthening, though manifested in fine-grained phonetic details, is not simply a low-level phonetic event but a complex linguistic phenomenon, closely linked to the enhancement of phonological features and positional strength that may license phonological contrasts. (C) 2005 Acoustical Society of America. [DOI: 10.1121/1.1861893]
\end{abstract}

PACS numbers: 43.70.Aj, 43.70.Eq [AL]

Pages: $3867-3878$

\section{INTRODUCTION}

One of the fundamental presuppositions shared among researchers in the fields of prosodic phonology and the phonetics-prosody interface is that speech utterances are produced in a "hierarchically organized structure of phonologically defined constituents and heads" (Beckman, 1996) with higher constituents being decomposed into lower constituents. The prosodic structure has been considered as an essential element in speech production, as it is not only a source of subphonemic variation, but also it constrains the phonological shape of a segment by forming different domains of application of phonological rules (cf. Selkirk, 1986; Jun, 1998). The present study explores how prosodic structure influences the production of the English vowels /a,i/, and how prosodic strengthening (i.e., temporal and/or spatial expansion of articulation due to accent and/or prosodic boundaries) can be related to the enhancement of phonological vowel features such as sonority and place features, which may ultimately shed light on the relationship between prosodic strengthening and linguistic contrast.

There are various approaches to determining prosodic groupings [see Shattuck-Hufnagel and Turk (1996) and Jun (1998) for a general review and a discussion]. One line of research assumes that the prosodic structure of an utterance is a grammatical entity in its own right, and it is realized on the surface by distinctive phonetic patterns, primarily via suprasegmental features such as pitch and temporal structure (Beckman and Pierrehumbert, 1986; Pierrehumbert and

${ }^{\text {a)} E l e c t r o n i c ~ m a i l: ~ t c h o @ ~ h a n y a n g . a c . k r ~}$
Beckman, 1988; Jun, 1993, and Beckman, 1996). The model of prosodic structure in this approach generally assumes that lower domains (e.g., Prosodic Words) are grouped into immediately higher levels [e.g., the Intermediate Phrases (ip)], eventually forming the Intonational Phrase (IP), the highest phonological constituent.

In seeking the phonetic correlates of prosodic structure, researchers have traditionally paid special attention to the end of prosodic domains. For example, prosodic units larger than a (phonological) word are generally defined in terms of the distribution of phrase-final intonational contrasts (e.g., Bolinger, 1970; Pierrehumbert, 1980; Pierrehumbert and Beckman, 1988) and final lengthening (e.g., Edwards et al., 1991; Beckman et al., 1992; Wightman et al., 1992). As for other phonetic events at the end of prosodic domains, it has been suggested that domain-final vowels may be produced with greater articulatory magnitude, resulting in greater V-to-C displacement (Fougeron and Keating, 1997; Cho, 2002; in-press; Tabain, 2003). Moreover, Cho (2004) has shown that boundary strength is reflected in cumulative $\mathrm{V}$-to-V coarticulatory resistance: domain-final vowels resist coarticulation with the following vowels to a greater degree across a higher prosodic boundary than across a lower one. These studies suggest that lengthening is just one of many phonetic phenomena occurring at the ends of phrases, which, taken together, may be thought of as a type of prosodic strengthening to mark the ends of prosodic domains. [See Byrd and Saltzman (2003) for simulations in the framework of the mass-spring gestural model, which demonstrated that a clock-slowing implementation may induce both temporal and spatial expansion at a stronger prosodic boundary.] 
Other recent studies, however, have shown that prosodic domains may also be signaled by extreme articulation at the left edges of the domains. For instance, consonants at the beginnings of phrases are more constricted than consonants in the middle of phrases. This phenomenon, known as domain-initial strengthening, has been documented in a series of electropalatographic studies in English, French, Korean, and Taiwanese (Fougeron and Keating, 1997; Cho and Keating, 2001; Fougeron, 2001; see also Keating et al. (1999) for a cross-linguistic comparison). (See also Cho and Jun, 2000 and Cho and McQueen, in press, for differential phonetic effects of domain-initial strengthening in connection with language-specific phonetic feature systems, in Korean and Dutch, respectively.) Domain-initial strengthening on a word level has also been observed acoustically by Turk and Shattuck-Hufnagel (2000).

Finally, prosodic strengthening effects can also be found in accented syllables, which may be thought of as the heads of Intermediate Phrases (see Beckman and Edwards, 1994). In addition to receiving distinctive tonal properties (such as pitch accents), segments in accented syllables are also more strongly articulated (e.g., Beckman et al., 1992; de Jong, 1995a, b; Erickson, 2002; Cho, in press).

These three strong prosodic positions (i.e., domain-final, domain-initial, and accented positions), all of which are subject to prosodic strengthening, are the loci of the present investigation: it examines the phonetic realization of the vowels /a,i/ both acoustically (as reflected in the F1-F2 vowel space) and articulatorily (as reflected in the maxima of the tongue, the jaw, and the lip opening). (Note that the jaw and lip opening maxima are likely to represent the degree of the vocal track opening that is related to sonority expansion, whereas the tongue maxima together with the acoustic data can be interpreted primarily as an index of how place features are phonetically realized).

While segments in prosodically strong positions are generally taken to be characterized by distinct phonetic patterns, most previous studies discussed above have focused on just one articulator or one or two prosodic positions, leaving it not entirely clear whether the phonetic signatures of prosodic structure differ in kind depending on the prosodic position. This leads to questions regarding whether and how articulation under accent differs from articulation at the edges of prosodic domains, and whether and how domain-initial articulation differs from domain-final articulation.

The second question is related to prosodic strengthening and linguistic contrast. Although prosodically strong positions have been regarded as "prominent" or "privileged" positions in which sonority is expanded (e.g., Beckman et al., 1992) and phonemic contrasts are likely to be maximized (e.g., de Jong, 1995a, b) or maintained (e.g., Beckman, 1998; Barnes, 2002; cf. Steriade, 1999), our knowledge on the relationship between the actual phonetic consequences of prosodic strengthening and linguistic contrast has been extremely limited. The present study therefore sets out to determine how prosodic strengthening is related to linguistic contrast. Specifically, it investigates how prosodic strengthening associated with different prosodic positions is related to the enhancement of phonological features of the vowels /a,i/, which could result in linguistic contrast being maximized, but possibly differentially depending on the source of prominence (e.g., in accented syllables versus at edges of prosodic domains; domain initially versus domain finally). Below are some specific hypotheses to be assessed in connection with the two main research questions outlined above.

With respect to accent-induced prominence, Edwards and Beckman (1988) and Beckman et al. (1992) proposed that an accent has the effect of enhancing a segment's intrinsic sonority (the Sonority Expansion Hypothesis). Under this account, the influence of accent on vowels is to make them more vowel-like by opening the vocal tract more; the influence on consonants is to make them more consonant-like by closing the vocal tract more tightly. Subsequently, de Jong (1995a) proposed that stress leads to hyperarticulation, enhancing the distinctive features of segments in a way that may maximize lexical distinctions, and sonority is just one of many distinctive features that are enhanced. In support of this localized hyperarticulation hypothesis, he showed that the tongue position for an English vowel / $\mho /$ is backer, being accompanied by a lower jaw position, ${ }^{1}$ which indicates both sonority expansion (jaw lowering) and the enhancement of place features ([+back] by the tongue backing).

Under this hypothesis, it is of particular interest to examine /a/ and /i/ at the same time because tongue lowering for $/ \mathrm{a} /$ could be interpreted as suggesting an enhancement of both nonsonority (place) feature (e.g., [+low]) and sonority feature, whereas tongue raising for $/ \mathrm{i} /$ could be interpreted as an enhancement of the place feature [+high], but in conflict with enhancement of the sonority feature as it reduces the size of the vocal track opening. In the latter case, however, sonority expansion may still be attained by making the jaw and the lip openings larger independently of the tongue raising. Thus, under the hyperarticulation hypothesis, it is predicted that the tongue is involved primarily in the enhancement of the nonsonority (place) features, whereas the jaw and the lips are involved primarily to achieve sonority feature enhancement, independently of the tongue.

With respect to boundary-induced prominence, Farnetani and Vayra (1996) hypothesize that while prominence under accent fits the localized hyperarticulation hypothesis, prominence at edges fits sonority expansion, which would heighten $\sharp \mathrm{CV}$ or $\mathrm{V} \# \mathrm{C}$ contrast (where $\#=$ some prosodic boundary). Thus, one can predict that prosodic strengthening at the edges of prosodic domains is different in kind from that associated with an accent, such that the former is driven primarily by the sonority expansion, but not by the enhancement of place features. If this were the case, all three articulators would converge on sonority expansion to be reflected in increased lip/jaw opening and tongue lowering for both vowels /a/ or /i/ at a higher prosodic boundary. It should be noted, however, that place features may still be opportunistically enhanced to the extent that is not in conflict with sonority expansion. The enhancement of the feature [+high] for /i/ (as may be reflected in tongue raising together with decreased lip and jaw openings) would be suppressed, as it would result in affecting the sonority expansion antagonistically. On the other hand, the enhancement of the place feature $[+$ low $]$ for $/ \mathrm{a} /$ goes hand in hand with sonority expan- 
sion: the articulatory maneuvers for sonority expansion of /a/ (e.g., increased lip and jaw openings and the tongue lowering) would effectively result in the enhancement of [+low].

This hypothesis will be tested separately for domaininitial and domain-final vowels since there have been different predictions made in the literature. For example, Fougeron and Keating (1997) speculate that strengthening may be very local to the domain edge such that the boundary-induced strengthening effect may be evident primarily in the domainfinal vowel and the domain-initial consonant, resulting in a greater V-to-C displacement across a larger prosodic boundary. Since a domain-initial vowel in $\# \mathrm{CV}$ is not strictly domain-initial, the sonority expansion effect might be smaller, or even absent, for domain-initial vowels.

In a traditional phonological framework (e.g., SPE, Chomsky and Halle 1968), vowels have often been defined in terms of the height and backness of the tongue (e.g., [+high, -back] for /i/ and [+low, + back] for /a/). However, some researchers have proposed that vowel features may be better understood in acoustic/auditory terms, for example in a F1-F2 plane (e.g., Neary, 1977, Flemming, 1995, Ladefoged, 2000). However, it should be noted here that in some other frameworks, vowels are described in terms of constriction degree and location (Wood, 1979, 1982; Jackson, 1988; cf. Browman and Goldstein, 1992). In this framework, /i/ is characterized as having a narrow constriction in the region of the hard palate and /a/ as having a narrow constriction in the lower pharynx. As described below, the articulatory component of the present study has been designed to identify the tongue position in a maxilla-based coordinate system defined by the occlusal plane without making specific reference to the opposing walls. Thus, it will be difficult to evaluate the prosodic strengthening effect on articulation in terms of the constriction degree and location. Similarly, the articulatory data in the present study provide positions of pellets attached on the tongue dorsum area, but not the tongue root area, which allows for only indirect assessment of the tongue's backness for $/ \mathrm{a} /$. Thus, the articulatory data with respect to the tongue's physical backness in this study must be interpreted as being indicative rather than conclusive.

\section{EXPERIMENT}

\section{A. Speech material and speakers}

Each sentence in the corpus included two test syllables (domain-final/domain-initial), yielding a $\mathrm{C}_{1} \mathrm{~V}_{1} \sharp \mathrm{C}_{2} \mathrm{~V}_{2}$ sequence ( $\#=$ some prosodic boundary) across two English words, as seen in sample sentences given in Table I. $\mathrm{C}_{1}$ and $\mathrm{C}_{2}$ were always $/ \mathrm{b} /$, whose articulation is known to minimally interfere with the vocalic lingual articulation, and $\mathrm{V}_{1}$ and $\mathrm{V}_{2}$ were homorganic (/i $\# \mathrm{bi} /$ and $/ \mathrm{a} \sharp \mathrm{ba} /$ ). As for prosodic variables, the boundary between the test syllables was varied from the Intonational Phrase boundary (IP), to the Intermediate Phrase boundary (ip), to the Word boundary $(\mathrm{Wd})$. Accentuation was also manipulated in preboundary and postboundary syllables, resulting in four pairs: ACC\#ACC, ACC\#UNACC, UNACC\#ACC, UNACC\#UNACC. Such a manipulation of prosodic factors yields three prosodic variables: (a) strength of prosodic boundary; (b) accentuation of
TABLE I. Sample sentences containing the sequence /ba $\sharp b a /$ with different prosodic boundaries (IP,ip,Wd) and accentual patterns. The words receiving accent are highlighted in bold.

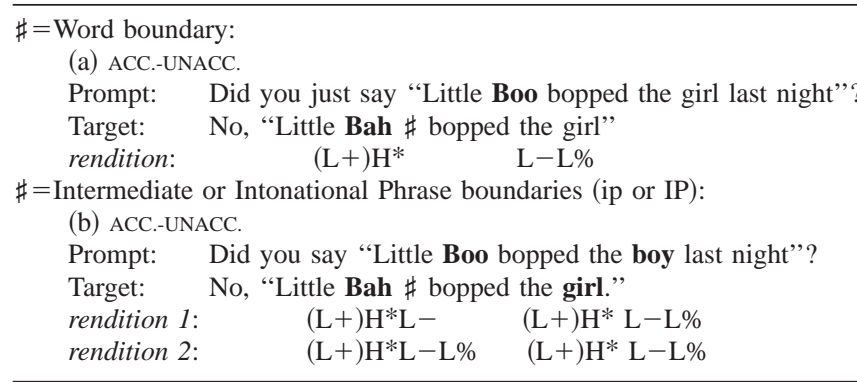

syllables adjacent to the boundary (accented, unaccented); (c) position-in-domain of test syllables (initial, final). This yielded a total of 48 different sequences (3 prosodic boundaries $\times 2$ accentual patterns $\times 2$ accentual patterns in the neighboring vowel $\times 2$ edges $\times 2$ vowel types).

Six native speakers of American English participated in the experiment, but only five were included in this study due to technical errors (see below). Participants were either linguistics students or postdoctoral fellows at UCLA. In order to control for the variation in rounding in the low vowel, speakers whose dialect lacked the phoneme / $/$ / were chosen, so that the vowel in the test word bop was produced as unrounded low back vowel /a/. Speakers were all trained in the production of English sentences in the ToBI (Tone and Break Index) framework (see Silverman et al., 1992; Beckman and Ayers, 1997) prior to the experiment. Before the actual recording date, each speaker participated in an approximately two-hour long practice session.

\section{B. Procedures}

Accent patterns were recorded in lexically contrasting discourse frames, as in Table I, in which the two word target sequence is /ba $\sharp \mathrm{ba} /$ in Little Bah bopped the girl. (/bi \#bi/ sequence tokens were produced in similar discourse frames as in Donna B. beeped at him.) The words highlighted in bold in the table received the accent. The prompt was read silently by the speaker to cue the intended accent patterns, which were provided using partial ToBI transcriptions in the script (see below). For a balanced number of tokens for ip and IP, speakers were asked to produce two different renditions for each sentence designed for inducing phrasal boundaries as shown in (b) in Table I: one with an ip boundary (rendition 1) and one with and IP boundary (rendition 2). Speakers had no difficulty producing two versions of each sentence (ip and IP), given the practice session and previous phonetic training. Each sentence was read twice in succession at their comfortable rate of speech and the entire list was read twice, for a total of four repetitions per sentence. This yielded a total of 960 sentence tokens for analysis (48 sentence types $\times 5$ speakers $\times 4$ repetitions).

An EMA system (Carstens Articulograph AG 100) was used with seven transducer coils to track articulatory movements (cf. Hoole, 1996). Two reference transducers were placed on the nose and upper gumline, or maxillary incisor, both of which were used by the EMA analyzing software to 
establish a maxilla-based coordinate system and to correct for head movement inside the helmet. The remaining five transducers were located on articulators: Two transducer coils were placed on the tongue: one at the tongue dorsum (TD) about $5 \mathrm{~cm}$ from the tongue tip and one at the tongue midsection (TM), about $2.5 \mathrm{~cm}$ from the tongue tip.

Next, in order to align the articulatory space to the maxillary occlusal plane, a flat plastic bite plate (approximately the size of bank card, custom-fit to the size of the subject's mouth) was used with two transducer coils attached along the midline that corresponds to the midsagittal line. The articulatory space was rotated so that the $x$ axis was the maxillary occlusal plane. Based on such a rotation, a maxillabased coordinate system was established in which the $x$ axis is parallel to the maxillary occlusal plane with the $y$ axis being perpendicular to that at the junction of the occlusal plane and the central maxillary incisor, and this is consistent across speakers. [A similar data processing procedure was employed by Tabain (2003); see Westbury (1994) for a further discussion about the usefulness of the occlusal plane.] The EMA data were sampled at $500 \mathrm{~Hz}$. The obtained kinematic signals were then submitted to low-pass filtering with a filter cutoff of $50 \mathrm{~Hz}$, using Tailor (Carstens' data processing program; see http://linguistics.ucla.edu/faciliti/facilities/ physiology/ema.htm.

The relevant $\mathrm{C}_{1} \mathrm{~V}_{1} \# \mathrm{C}_{2} \mathrm{~V}_{2}$ portion of the audio recording was transcribed, with the aid of an acoustic display, by two trained ToBI transcribers (one the author) following the criteria set forth in the ToBI transcription system. In general, accents receive either $\mathrm{H}^{*}$ or $\mathrm{L}+\mathrm{H}^{*}$, and three prosodic boundaries were identified: the IP boundary (marked by a boundary tone and a break index 4); the ip boundary (marked by a break index 3 , a phrasal tone, and no boundary tone); the Word boundary (marked by a break index 1, in the middle of an Intermediate Phrase). The two transcribers identified identical locations of accent in every token of the entire dataset. The only difference between the two transcribers came from a choice between the Intonational Phrase boundary and the Intermediate Phrase boundary, and between $\mathrm{L}+\mathrm{H}^{*}$ and $\mathrm{H}^{*}$ for accented words. Because the difference between IP and ip boundaries is an important experimental variable in this study, only tokens whose renditions were agreed on by the two transcribers $(94.3 \%)$ were used for analysis. ${ }^{2}$

The extreme points of the tongue mid (TM) and tongue dorsum (TD) transducers were primarily identified from minima in the tangential velocity signal, following Löfqvist, Gracco, and Nye (1993) and Löfqvist (1999). These points were cross-checked by inspecting a sagittal display of the tongue movement trajectories, in which "turn-around" points could be found. In the usual case, these correspond to the tangential velocity minima. However, cross-checking was especially useful when there was more than one tangential velocity minimum or when there was a temporal offset between the $x$ and $y$ maxima. In such cases, as suggested by a reviewer, the maxima in the $x$ and $y$ dimensions were taken separately at different times to record the actual extreme $x$ and $y$ position values during vowel articulation. Out of 960 tokens, 247 tokens $(25.7 \%)$ showed $x-y$ timing misalign- ment, leading to a mean $\pm 8.9 \mathrm{~ms}$ temporal offset for the entire tokens.

The measurements for the tongue midsection and dorsum transducers were always taken at the same point in time. In most cases, the tangential velocity minima for the two transducers were synchronized. But, for the sake of consistency, the dorsum maxima were taken in synchrony with the midsection maxima. The extraction of the extreme points for the jaw during vowels was performed by taking the maximum Euclidean distance between the transducer at the lower gumline and the transducer at the upper gumline (reference point). Similarly, the extreme points for the lips during vowels were taken from the lip aperture profile as the maximum Euclidean distance between the upper and lower lip transducers. The jaw and lip opening measurements were aligned in time. Finally, the formant values were obtained from the acoustic signals sampled at $16000 \mathrm{~Hz}$ in synchrony with tongue maximum points. F1 and F2 were determined from the LPC spectra (with 18 or 20 coefficients) superimposed on FFT spectra with a 512-point $(32 \mathrm{~ms})$ frame window centered around the tongue maximum points.

Statistical evaluation of the systematic influence of prosodic factors was primarily based on repeated measures (RM) Analyses of Variance. The within-subject factors considered were Prosodic Boundary (IP,ip,Wd) and Accent (ACC, UNACC). The factor Speaker was added as a betweensubject factor to the analysis in order to take into account the speakers' contribution to any observed significance effect. Thus, a significant interaction between a prosodic factor and the factor Speaker would imply speaker-by-speaker differences. Therefore, whenever there is such a significant interaction, remarks on each speaker behavior will be made, in comparison with the overall pattern across speakers. However, adding the between-subject factor Speaker requires that each speaker contributes multiple scores per condition, making it impractical to average the data over repetitions. To compensate for this, the alpha level for significance was set more conservatively at $1 \%(p<0.01)$. Finally, to avoid violating the sphericity assumption, Huynh-Feldt corrected degrees of freedom (thus, often fractional values) were used in generating $\mathrm{F}$ ratio and $p$ values.

\section{RESULTS}

\section{A. Domain-final vowels $\left(\mathrm{V}_{1} \#\right)$}

\section{Effect of accent on $\mathrm{V}_{1}$}

a. Accent effect on $\mathrm{V}_{1} F 1$ and $F 2$ and tongue maxima The results are summarized in Fig. 1. Before delving into the detailed results, a few notes regarding the figure formats are in order. Each value in the figure (and other figures below) is the mean of speaker means with data pooled across other conditions (in this case, Boundary Type and Accent of the adjacent vowel). The F1-F2 formant plots shown in Fig. 1(a) and elsewhere have been drawn with the UCLA PlotFormants software. The scales are in accordance with the Bark scale, and the ellipses are drawn with radii of two standard deviations of the raw data along the axes of the first two principal components. 
ACCENT EFFECTS: PRE-BOUNDARY VOWELS

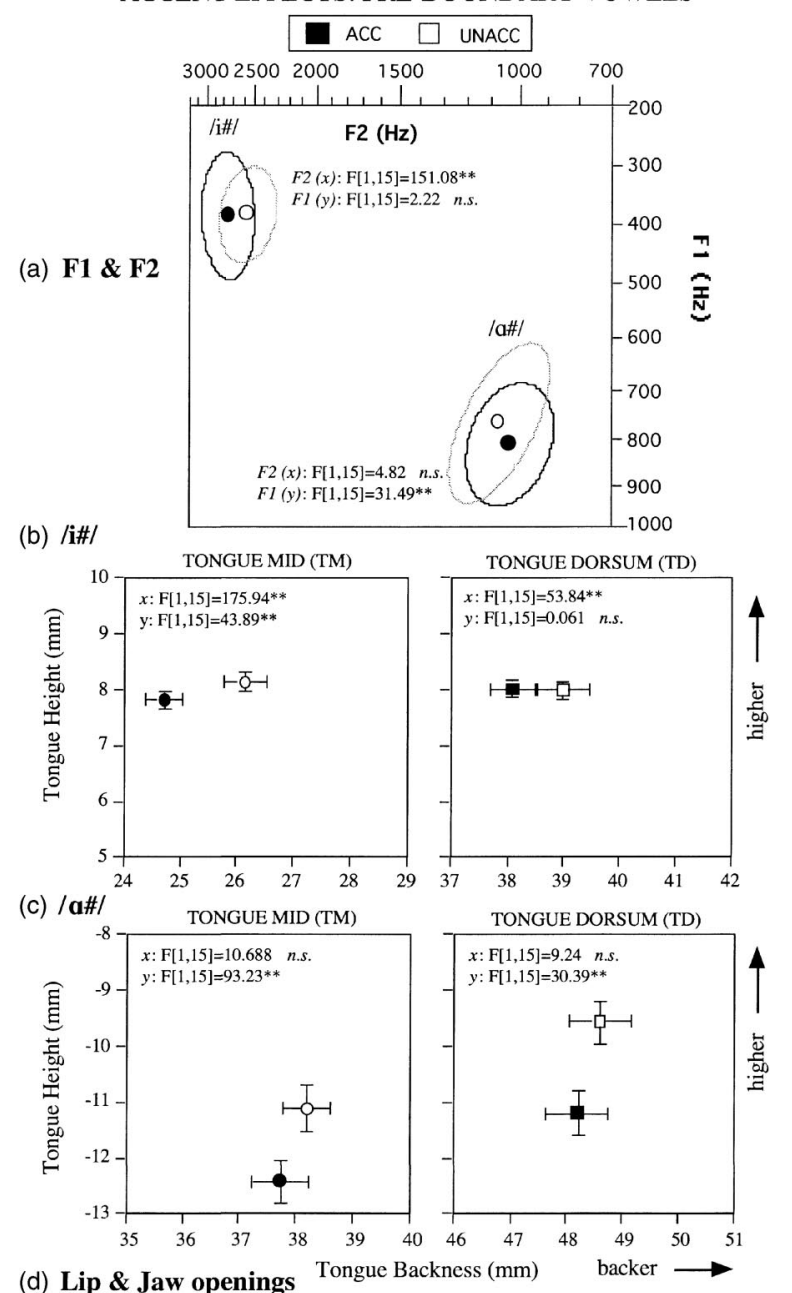

(d) Lip \& Jaw openings
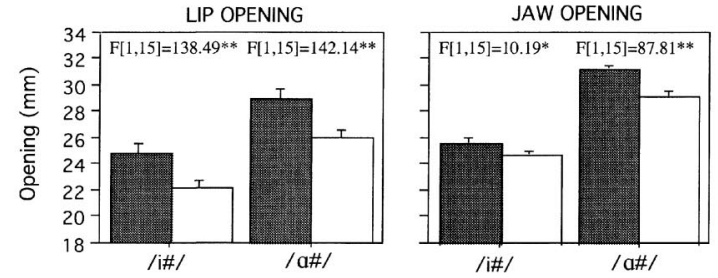

FIG. 1. Effect of Accent on F1 and F2 (a), the tongue maxima /i $\# /$ and /a \#/ (b), (c), and the lip and jaw opening maxima (d) in the domain-final position. (* refers to $p<0.01$; ** refers to $p<0.001 ; N=30$.) Note that each value in the figure (and other figures below) is the mean of speaker means with data pooled across other conditions (in this case, across prosodic boundary conditions and accent conditions of the adjacent domain-initial vowel). Thus, $N$ is 30 ( 5 speakers $\times 3$ prosodic boundaries $\times 2$ accent conditions of the adjacent vowel).

Now consider /i \#/. Results of RM ANOVAs showed that /i\#/ was significantly fronter when accented versus unaccented in both the acoustic (F1-F2) and the articulatory vowel spaces [Figs. 1(a), 1(b)]. There were no interactions with Speaker (showing consistency across speakers).

In the vertical dimension, however, there was no main accent effect on /i $\# /$ in either the acoustic or the articulatory vowel space [Figs. 1(a), 1(b)]. However, there were significant interactions with Speaker $(\mathrm{F}[8,30]=32.12, p<0.001$ for $\mathrm{F} 1 ; \mathrm{F}[8,30]=8.21, p<0.01$ for TM; $\mathrm{F}[8,30]=6.39, p<0.01$ for TD). Two speakers showed lowering effects [S1, in both the acoustic (F1) and the articulatory (TM) dimensions; S2, only in the articulatory (TD) dimension]. On the other hand, two other speakers $(\mathrm{S} 4, \mathrm{~S} 5)$ showed the opposite pattern (i.e., raising) in the acoustic (F1) dimension, but not in the corresponding articulatory dimension.

For $/ \mathrm{a} \sharp /$, there were reliable acoustic and articulatory lowering effects when accented [Figs. 1(a), 1(c)] with no interactions with the Speaker. Contrary to the robust lowering effect, however, in the horizontal dimension /a $\sharp$ / showed no consistent backing effect [Figs. 1(a), 1(c)]. The backing effect was found in two speakers $(\mathrm{S} 1, \mathrm{~S} 3)$ only in the acoustic dimension (F2), while no speakers showed corresponding backing in the articulatory dimension.

In summary, the high/front / $i \sharp /$, when accented, is consistently fronter in both acoustic and articulatory vowel spaces but not necessarily higher: Only two speakers $(\mathrm{S} 1, \mathrm{~S} 2)$ showed accent-induced raising in both acoustic and articulatory dimensions and two others $(\mathrm{S} 4, \mathrm{~S} 5)$ showed the opposite in the acoustic dimension. The low/back vowel /a $\# /$ is consistently lower when accented, but backer in two speakers $(\mathrm{S} 1, \mathrm{~S} 3)$ only in the acoustic dimension (F2). (See Sec. IV A for a discussion on this).

\section{b. Accent effect on $\mathrm{V} 1$ lip and jaw opening maxima} Both lip and jaw opening maxima were reliably larger when accented than unaccented regardless of the vowel type [Fig. 1(d)]. There were no interactions with the Speaker, showing a consistent pattern across speakers.

\section{Effect of boundary type on $\mathbf{v}_{1}$}

a. Boundary effect on F1 and F2 and tongue maxima in $\mathrm{V}_{1}$ For $/ \mathrm{i} \# /$, there were significant accent-induced raising effects [Figs. 2(a), 2(b)]. /i\#/ was higher before a higher boundary in both acoustic (F1) and articulatory (tongue height) dimensions ( $\mathrm{IP}=\mathrm{ip}>\mathrm{Wd}, p<0.01)$. There were no significant interactions with the Speaker. [All five speakers showed either a two-way (IP $>\mathrm{Wd}$ or ip $>\mathrm{Wd}$ or IP $>$ ip) or a three-way (IP $>$ ip $>$ Wd) distinction.]

As for the fronting effects, inconsistency was found between the acoustic (F2) and the articulatory $(x)$ dimensions [Figs. 2(a), 2(b)]. There was a significant fronting effect in $\mathrm{F} 2$ before a higher boundary ( $\mathrm{IP}=\mathrm{ip}>\mathrm{Wd}, p<0.01$ ), with no interaction with the Speaker. On the other hand, in the articulatory $(x)$ dimension, there were no main effects but significant interactions with Speaker $(\mathrm{F}[8,30]=12.23, p<0.001$ for $\mathrm{TM} ; \mathrm{F}[8,30]=9.39, p<0.01$ for $\mathrm{TD})$ such that only one speaker (S5) showed a significant fronting effect in TM.

For $/ \mathrm{a} \sharp /$, there was significant lowering and backing in both the acoustic and the articulatory vowel spaces [Figs. 2(a), 2(c)]. In the acoustic space, boundary-induced lowering and backing of /a $\# /$ was found in three speakers (S1, S2, S5 for lowering; S3, S4, S5 for backing). In the articulatory dimension, the lowering and backing effect becomes even clearer: four speakers (excluding S1) showed tongue lowering in either TM or TD and all five showed tongue backing before a higher prosodic boundary in both TD and TM.

In summary, in the vertical dimension, there was a consistent boundary-induced raising effect for /i $\# /$ in both acoustic and articulatory spaces; in the horizontal dimension, a fronting effect for / $\mathrm{i} \# /$ was found primarily in the acoustic dimension. (See Sec. IV B for a discussion on this.) These 
BOUNDARY EFFECTS: PRE-BOUNDARY VOWELS

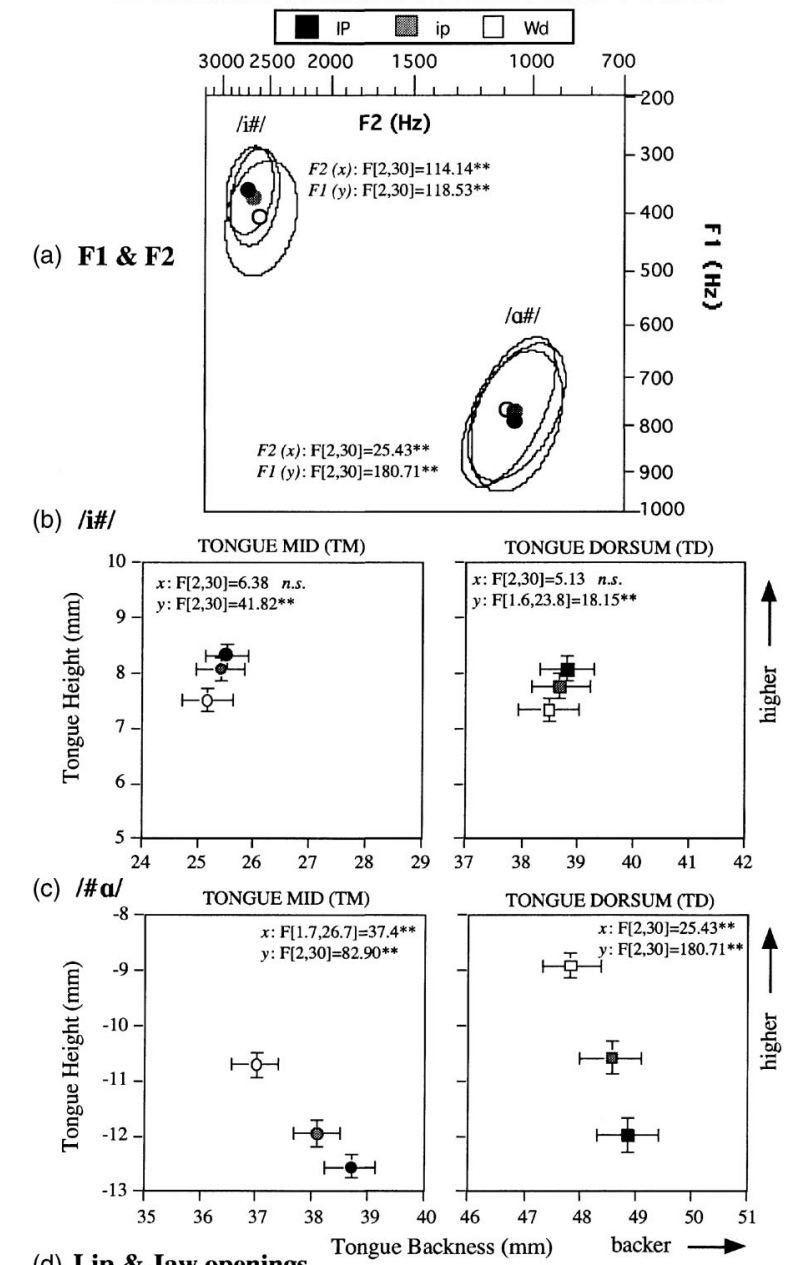

(d) Lip \& Jaw openings
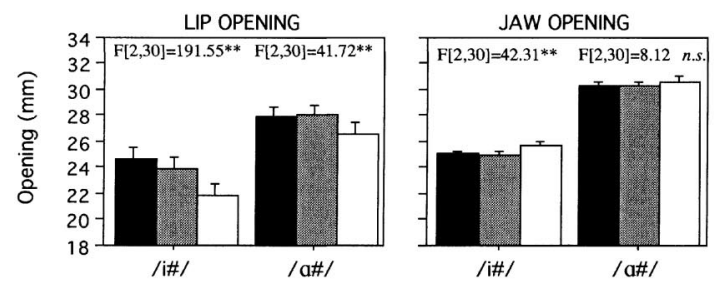

FIG. 2. Effect of Prosodic Boundary on F1 and F2 (a), the tongue maxima $/ \mathrm{i} \# /$ and $/ \mathrm{a} \sharp /(\mathrm{b}),(\mathrm{c})$, and the lip and jaw opening maxima (d) in the domainfinal position. (* refers to $p<0.01$; ** refers to $p<0.001 ; N=20$.) Note that in this case, $N$ is $20(5$ speakers $\times 2$ accent conditions $\times 2$ accent conditions of the adjacent vowel.)

boundary effects on /i $\sharp /$ differed from the accent effects in that the former induced consistent acoustic/articulatory raising whereas the latter induced consistent acoustic/ articulatory fronting. For the low/back vowel /a $\sharp /$, there were consistent boundary-induced lowering and backing in both the acoustic and the articulatory vowel spaces. In particular, the boundary effect on backing of /a $\# /$ was more consistent than was the accent effect, while both the boundary and the accent effects showed similar acoustic/ articulatory lowering of /a $\sharp /$.

b. Boundary effect on lip and jaw opening maxima in $\mathrm{V}_{1}$

As shown in Fig. 2(d), the lip opening was significantly larger for both vowels before a higher prosodic boundary ( $\mathrm{IP}=\mathrm{ip}>\mathrm{Wd}, p<0.01)$. There were no interactions with the Speaker. In contrast to the lip opening pattern, there was a symmetry in the jaw opening maxima between /i $\# /$ and $/ a \sharp /$. On the one hand, as shown in the right panel of Fig. 2(d), /i\#/ showed a small but significantly smaller jaw opening before a higher boundary (ip or IP) than before a lower one $(\mathrm{Wd})(p<0.01)$, with no significant interaction with the Speaker. (All five speakers showed the same pattern.) On the other hand, /a\#/ showed no main effect on the jaw opening but a significant interaction with the Speaker $(\mathrm{F}[8,30]=11.98, p<0.01)$. Notably, only one speaker (S5) showed an increase in the jaw opening at a higher boundary and two speakers $(\mathrm{S} 2, \mathrm{~S} 4)$ showed a decrease in the jaw opening at a higher boundary, as was the case for /i $\sharp /$.

In summary, domain-final vowels were produced with a larger lip opening before higher prosodic boundaries, but not necessarily with a larger jaw opening. In fact, for /i \#/, the jaw opening was reliably smaller at a higher boundary, as opposed to the accent-induced increase in the jaw opening for /i\#/. (See Sec. IV B for a relevant discussion on the discrepancy between the jaw and the lip openings.)

\section{B. Articulatory maxima in domain-initial (post-boundary) vowels $\left(\# \mathrm{CV}_{2}\right)$}

\section{Effect of accent on $\mathrm{C}_{2} \mathrm{~V}_{2}$}

a. Accent effect on F1 and F2 and tongue maxima in $\mathrm{C}_{2} \mathrm{~V}_{2} \quad \mathrm{For} / \sharp \mathrm{bi} /$, there were consistent fronting effects, with no interactions with the Speaker, in both the acoustic and the articulatory dimensions [Figs. 3(a), 3(b)]. However, as was the case for the domain-final accent effect, there were unexpected accent-induced lowering effects in both the acoustic and articulatory dimensions; for the latter the effect was evident in TM. Only one speaker (S4) showed accent-induced raising for /i/ in both the acoustic and the articulatory vowel space. Conversely, four speakers (S1,S2,S3,S5) showed lowering in the acoustic dimension (F1), with two speakers $(\mathrm{S} 1, \mathrm{~S} 5)$ also showing lowering in the articulatory dimension.

For / $\#$ ba/, accent entailed significant lowering and backing in the acoustic dimension with no interaction with the Speaker [Fig. 3(a)]. The articulatory data showed a corresponding tongue lowering effect [Fig. 3(c)], but not a corresponding backing effect. Only one speaker (S3) showed significant tongue backing and two $(\mathrm{S} 2, \mathrm{~S} 4)$ tongue fronting. (See Sec. IV A for possible explanations for the discrepancy between the acoustic and the articulatory data.)

In summary, as was for domain-final vowels, in the backness dimension domain-initial /i/ was consistently fronter when accented in both acoustic and articulatory spaces, but in the vertical dimension, it was actually lower, at least in the acoustic dimension. The low/back /a/ was generally lower and backer when accented, although the backing effect for /a/ was primarily evident in the acoustic dimension.

b. Accent effect on V2 lip and jaw opening maxima As shown in Fig. 3(d), accented $V_{2}$ is associated with greater jaw and lip openings than unaccented $\mathrm{V}_{2}$, regardless of the vowel type. All speakers showed significantly greater jaw and lip opening patterns for accented domain-initial vowels. 


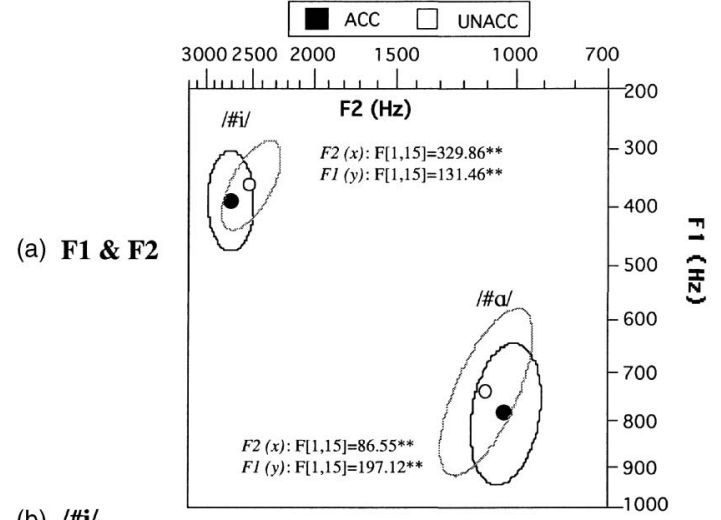

(b) /\#i/
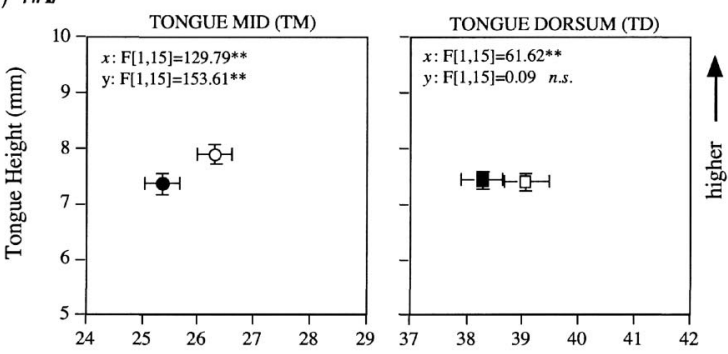

(c) $/ \# \mathbf{a} /$

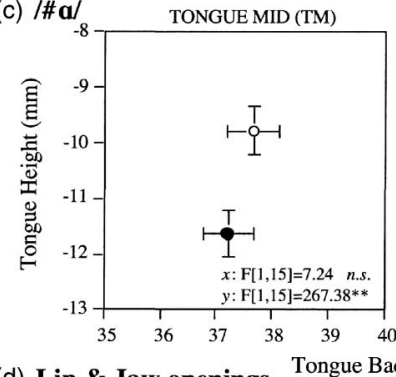

TONGUE DORSUM (TD)
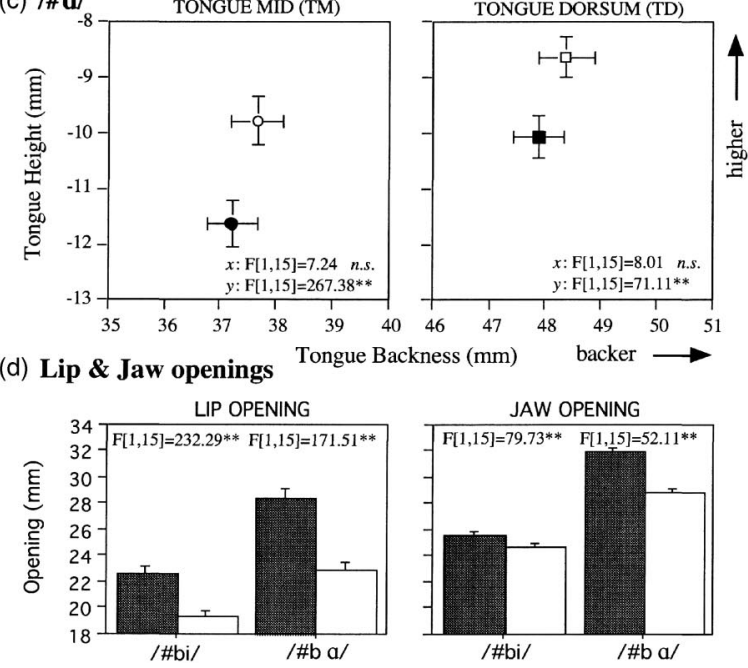

FIG. 3. Effect of Accent on F1 and F2 (a), the tongue maxima / $\# \mathrm{i} /$ and / $\# \mathrm{a} /$ (b), (c), and the lip and jaw opening maxima (d) in the domain-initial position. (* refers to $p<0.01$; $* *$ refers to $p<0.001 ; N=30$.)

\section{Effect of boundary type on domain-initial $\mathrm{C}_{2} \mathrm{~V}_{2}$}

a. Boundary effect on $\mathrm{C}_{2} \mathrm{~V}_{2} \mathrm{Fl}$ and F2 and tongue maxima The results with respect to the boundary effect on the domain-initial vowels are not as robust and consistent as the boundary effect on the domain-final vowels. Consider /\#bi/ in the acoustic F1-F2 vowel space [Fig. 4(a)]. There was no main effect of Boundary in the acoustic dimension, but significant interactions with Speaker $(\mathrm{F}[8,30]=5.77, p$ $<0.01$ in $\mathrm{F} 1 ; \mathrm{F}[8,30]=6.68, p<0.01$ in $\mathrm{F} 2$ ). Only one speaker (S5) showed boundary-induced raising and backing (not fronting) for /i/, and all others showed no significant differences. On the other hand, in the articulatory dimensions, there was significant tongue raising for $/ i \sharp /$ at a higher boundary (IP $>\mathrm{Wd}, p<0.01$ ), as reflected in TM in Fig. 4(b). [Four speakers $(\mathrm{S} 1, \mathrm{~S} 2, \mathrm{~S} 4, \mathrm{~S} 5)$ showed a articulatory raising effect at a higher boundary, and one speaker (S3) showed the opposite trend.] By contrast, there was no evidence for

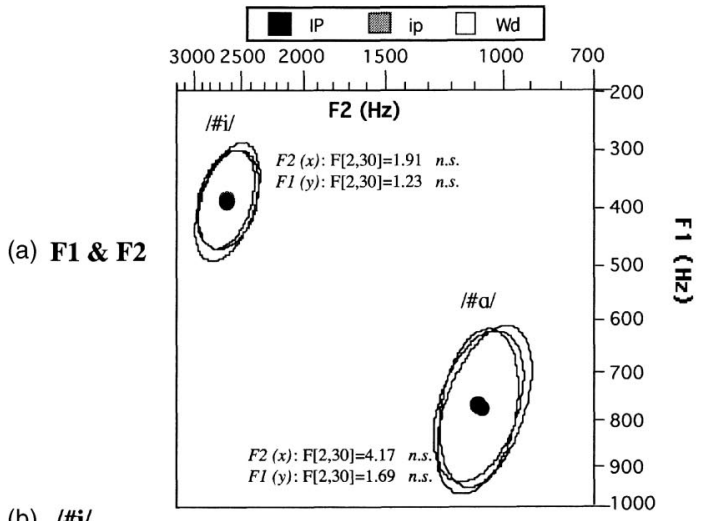

(b) /\#i/
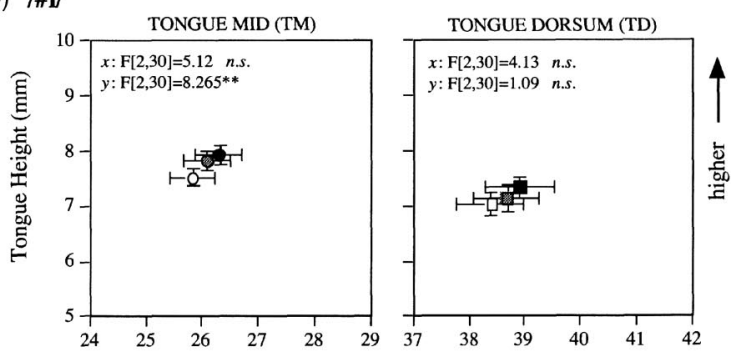

(c) $/ \# \mathbf{a} /$

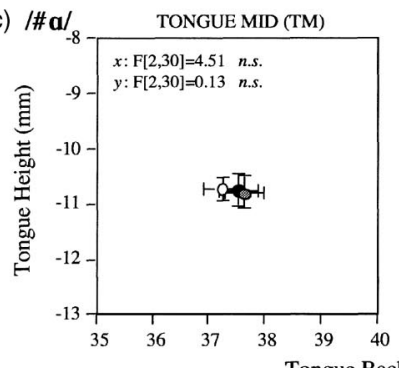

TONGUE DORSUM (TD)

(d) Lip \& Jaw openings Tongue Backness (mm)
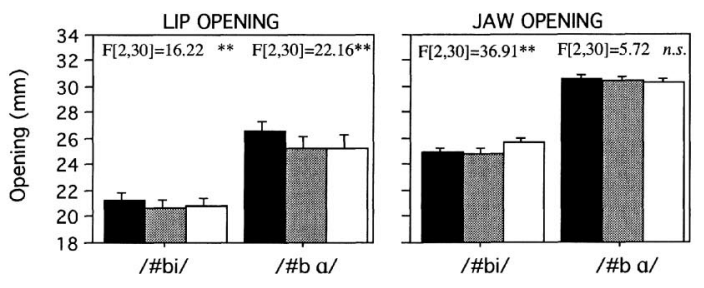

FIG. 4. Effect of Prosodic Boundary on F1 and F2 (a), the tongue maxima $/ \# \mathrm{i} /$ and $/ \# \mathrm{a} /(\mathrm{b}),(\mathrm{c})$, and the lip and jaw opening maxima (d) in the domaininitial position. (* refers to $p<0.01$;** refers to $p<0.001 ; N=20$.)

boundary-induced tongue fronting: only one speaker (S5) showed a tongue fronting effect but two others $(\mathrm{S} 3, \mathrm{~S} 4)$ showed the opposite.

Turning to / $\sharp$ ba/, as shown in Figs. 4(a), 4(c), both acoustic and articulatory data showed no main effect but significant interactions with the Speaker in all cases. In the backness dimension, two speakers $(\mathrm{S} 3, \mathrm{~S} 5)$ showed acoustic and articulatory backing for /a/ after a higher prosodic boundary, and an additional speaker (S4) showed articulatory backing only. In the height dimension, only one speaker (S1) showed the lowering of $/ \mathrm{a} /$ in the acoustic dimension and two $(\mathrm{S} 3, \mathrm{~S} 5)$ in the articulatory dimension. Interestingly, the two speakers $(\mathrm{S} 3, \mathrm{~S} 5)$ who showed tongue lowering were the ones who showed acoustic (and articulatory) backing for /a/.

In summary, domain-initial /i/ was higher at a higher boundary, more so in the articulatory than in the acoustic dimension. One speaker (S5) showed both acoustic and ar- 
ticulatory raising and three speakers $(\mathrm{S} 1, \mathrm{~S} 2, \mathrm{~S} 4)$ showed articulatory raising only. Unlike the domain-final /i/, however, there was no consistent evidence of either an acoustic or an articulatory fronting effect: only S5 showed a fronting effect only in the articulatory dimension. The effect of Boundary on domain-initial /a/ was less clear. Notably, however, two speakers $(\mathrm{S} 3, \mathrm{~S} 5)$ showed boundary-induced backing in both the acoustic and the articulatory dimensions, and the same two speakers also showed articulatory tongue lowering, which was not coupled with acoustic lowering. (See Sec. IV A for a discussion on this.)

b. Boundary effect on $\mathrm{C}_{2} \mathrm{~V}_{2}$ lip and jaw maxima There was a significant main effect of Boundary on the maximum lip opening for $/ \sharp$ bi/ $(I P>i p=W d, p<0.01)$ seen in Fig. 4(d). But there was a significant interaction with Speaker $(\mathrm{F}[8,30]=19.77, p<0.001)$ : two speakers $(\mathrm{S} 1, \mathrm{~S} 5)$ showed a significantly larger lip opening after a higher boundary $(\mathrm{IP} \geqslant \mathrm{ip}>\mathrm{Wd}$ ), while two others $(\mathrm{S} 2, \mathrm{~S} 4)$ showed nonsignificant trends in the same direction. The jaw opening data for $/ \sharp$ bi/ also showed a significant main effect, as shown in the right panel of Fig. 4(d), but in the opposite direction: the jaw opening was smaller for IP and ip than for Wd $(p<0.01)$. Four speakers indeed showed a decreased jaw opening at a higher boundary $(\mathrm{IP}=\mathrm{ip}<\mathrm{Wd}, p<0.01)$. (Recall that the same four speakers showed a boundary-induced decrease in the jaw opening for the domain-final /i $\# /$.)

As for $/ \sharp \mathrm{ba} /$, there was a main effect of Boundary, showing a significantly larger lip opening at a higher prosodic boundary (IP $>\mathrm{ip}=\mathrm{Wd}, p<0.01)$, as shown in Fig. 4(d). There was, however, a significant interaction with Speaker $(\mathrm{F}[8,30]=11.21, p<0.01)$ due to one speaker $(\mathrm{S} 3)$, who showed the opposite pattern. (Four speakers showed a similar pattern, but only significantly so for S1, S5). For the jaw opening for $/ \sharp \mathrm{ba} /$, there were no effects of Boundary.

In summary, for domain-initial /i/, the lip opening is generally larger at a higher prosodic boundary (found in four speakers) but the jaw opening is smaller (found in four speakers) at higher prosodic boundaries; for domain-initial /a/, the lip opening was generally larger at higher boundaries but there was no boundary effect at all on the jaw opening.

\section{SUMMARY AND DISCUSSION}

The results are recapitulated below and in Table II.

A summary of accent effects (when accented) are as follows: (1) Both the lip and the jaw openings for both /a/ vs /i/ are generally larger; (2) the high front vowel /i/ is consistently fronter but not necessarily higher (only two speakers showed raising effects only in the acoustic dimension); and (3) the low back vowel /a/ is generally lower, but backer only in the acoustic vowel space. ${ }^{5}$

A summary of boundary effects (at a higher boundary) are as follows: (1) The lip opening for both vowels /i,a/ is generally larger, but more consistently so in domain-final positions than in domain-initial ones; (2) the jaw opening is not larger. Instead, it is significantly and consistently smaller for $/ \mathrm{i} /$, while there is no consistent jaw opening pattern for /a/; (3) the high front vowel /i/ is consistently higher in domain-final positions. But in domain-initial positions, it is generally higher only in the articulatory vowel space; (4) the
TABLE II. A summary of the main effects of Accent and Boundary Type on domain-final (_\#) and domain-initial (\#_) vowels. Cells with descriptions indicate that the main effects are significant at $p<0.01$ with "*" and $p$ $<0.001$ with "**." When there are significant interactions between a prosodic factor and the factor Speaker, the individual speaker's contribution to the observed pattern is also noted parenthetically based on the results of a series of $t$-tests conducted between conditions with each speaker.

\begin{tabular}{|c|c|c|c|c|c|}
\hline \multirow{2}{*}{$\begin{array}{l}\mathrm{V}- \\
\text { type }\end{array}$} & \multirow{2}{*}{$\begin{array}{c}\text { Phonetic } \\
\text { dimension } \\
\text { s }\end{array}$} & \multicolumn{2}{|c|}{ Accent effect } & \multicolumn{2}{|c|}{ Boundary effect } \\
\hline & & _\# (V1) & $\#_{-}$(V2) & _\# (V1) & $\#_{-}$(V2) \\
\hline \multirow{15}{*}{ /i/ } & \multirow[t]{3}{*}{ F1 } & n.s. & n.s. & $* *$ & \multirow{3}{*}{$\begin{array}{l}n . s . \\
\text { H (S5) }\end{array}$} \\
\hline & & $\mathrm{H}(\mathrm{Ss} 4,5)$ & $\mathrm{H}(\mathrm{S} 2)$ & \multirow{2}{*}{$\mathrm{H}$} & \\
\hline & & $\mathrm{L}(\mathrm{S} 1)$ & $\mathrm{L}(\mathrm{Ss} 1,3,4,5)$ & & \\
\hline & \multirow[t]{3}{*}{ Tongue $y$} & & $* *$ & $* *$ & $* *$ \\
\hline & & $\mathrm{L}(\mathrm{Ss} 1,2)$ & $\mathrm{H}(\mathrm{S} 2)$ & $\mathrm{H}$ & $\mathrm{H}(\mathrm{Ss} 1,2,4,5)$ \\
\hline & & & $\mathrm{L}(\mathrm{Ss} 1,5)$ & & $\mathrm{L}(\mathrm{S} 3)$ \\
\hline & \multirow[t]{2}{*}{$\mathrm{F} 2$} & $* *$ & $* *$ & $* *$ & n.s. \\
\hline & & $\mathrm{F}$ & $\mathrm{F}$ & $\mathrm{F}$ & B (S5) \\
\hline & \multirow[t]{3}{*}{ Tongue $x$} & $* *$ & $* *$ & n.s. & n.s. \\
\hline & & $\mathrm{F}$ & $\mathrm{F}$ & F (S5) & $\mathrm{F}(\mathrm{S} 5)$ \\
\hline & & & & B $(\mathrm{Ss} 1,4)$ & $\mathrm{B}(\mathrm{Ss} 3,4)$ \\
\hline & \multirow[t]{2}{*}{ Lip } & $* *$ & $* *$ & ** & \\
\hline & & LG & LG & LG & LG $(\mathrm{Ss} 1,5)$ \\
\hline & \multirow[t]{2}{*}{ Jaw } & $* *$ & $* *$ & $* *$ & \\
\hline & & LG & LG & SM & $\mathrm{SM}(\mathrm{Ss} 1,2,3,5)$ \\
\hline \multirow{18}{*}{ /a/ } & \multirow[t]{3}{*}{$\mathrm{F} 1$} & $* *$ & $* *$ & ** & n.s. \\
\hline & & $\mathrm{L}$ & $\mathrm{L}$ & $\mathrm{L}(\mathrm{Ss} 1,2,5)$ & $\mathrm{L}(\mathrm{S} 1)$ \\
\hline & & & & & $\mathrm{H}(\mathrm{S} 2)$ \\
\hline & \multirow[t]{3}{*}{ Tongue $y$} & $* *$ & $* *$ & $* *$ & \multirow{3}{*}{$\begin{array}{l}\text { n.s. } \\
\mathrm{L}(\mathrm{Ss} 3,5) \\
\mathrm{H}(\mathrm{Ss} 2,4)\end{array}$} \\
\hline & & $\mathrm{L}$ & $\mathrm{L}$ & $\mathrm{L}$ & \\
\hline & & & & & \\
\hline & \multirow[t]{3}{*}{$\mathrm{F} 2$} & $* *$ & $* *$ & $* *$ & \multirow{3}{*}{$\begin{array}{l}\text { n.s. } \\
\mathrm{B}(\mathrm{Ss} 3,5) \\
\mathrm{F}(\mathrm{S} 1)\end{array}$} \\
\hline & & B $(\mathrm{Ss} 1,3)$ & B & B $(\mathrm{Ss} 3,4,5)$ & \\
\hline & & & & & \\
\hline & \multirow[t]{3}{*}{ Tongue $x$} & \multirow[t]{3}{*}{ n.s. } & n.s. & $* *$ & \\
\hline & & & $\mathrm{B}(\mathrm{S} 3)$ & B & \multirow{2}{*}{ B $(\mathrm{Ss} 3,4,5)$} \\
\hline & & & $\mathrm{F}(\mathrm{Ss} 2,4)$ & & \\
\hline & \multirow[t]{3}{*}{ Lip } & $* *$ & $* *$ & $* *$ & $* *$ \\
\hline & & LG & LG & LG & LG $(\mathrm{Ss} 1,5)$ \\
\hline & & & & & SM (S3) \\
\hline & \multirow[t]{3}{*}{ Jaw } & $* *$ & $* *$ & $* *$ & \multirow{3}{*}{ n.s. } \\
\hline & & LG & LG & LG (S5) & \\
\hline & & & & SM $(\mathrm{Ss} 2,4)$ & \\
\hline
\end{tabular}

Note: $\mathrm{H}=$ higher, $\mathrm{L}=$ lower, $\mathrm{F}=$ fronter, $\mathrm{B}=$ backer, $\mathrm{SM}=$ smaller, $\mathrm{LG}$ $=$ larger; Ss $=$ Speakers. Numbers in parentheses indicate speaker IDs.

high front vowel /i/ is consistently fronter in domain-final positions primarily in the acoustic space. In domain-initial positions, it is hardly fronter; (5) the low back vowel /a/ is consistently lower in domain-final positions. In domaininitial positions, there is no significant lowering effect for $/ \mathrm{a} /$; and (6) the low back vowel /a/ is consistently backer, though the effect is more consistently so in domain-final than in domain-initial position.

\section{A. Accent and featural enhancement}

It was hypothesized that under accent, the maximum position of the tongue should increase in a direction to enhance place features, whereas the jaw and lip openings should be expanded in order to increase sonority. As far as the opening of the vocal tract is concerned, the results showed that accented vowels are produced with an increase in jaw and lip openings for both vowels /a,i/, which is taken to be an enhancement of the sonority feature.

As for place features [high, low, back], defined in the acoustic F1-F2 vowel space, the results showed that $/ \mathrm{a} /$ is generally lower and backer when accented versus unac- 
cented, which can be interpreted as an enhancement of both [+low] and [+back] for /a/. For the high front vowel /i/, however, it is consistently fronter, but not necessarily higher, suggesting that accentuation induces an enhancement of [-back], but not a consistent enhancement of [+high] insofar as the features are defined in the acoustic vowel space. This suggests that not all nonsonority (place) features defined in the acoustic dimension are enhanced uniformly under accent.

Now, let us consider how the acoustic results can be linked to the articulatory results. For /a/, the acoustic lowering effect (enhancement of $[+$ low $]$ ) was further evident in the articulatory space. The enhancement of $[+$ low $]$ as reflected in the raised F1 may be attributable to the articulatory maneuvers involving not only the jaw and lip opening but also the tongue lowering, converging on sonority expansion (e.g., Harrington et al., 2000; Erickson, 2002). On the other hand, the enhancement of [+back] evident in backing in the acoustic space (lowered F2) was not consistently accompanied by the tongue backing in the articulatory space, (while the tongue lowering was observed in all speakers). In the present study, the articulatory vowel space reflects a Cartesian vowel space defined by the occlusal plane. The acoustic F2 lowering may then not be directly translatable in terms of the tongue backing in an articulatory space defined this way. Instead, as Wood $(1979,1982)$ proposed, the observed F2 lowering for / $\mathrm{a} /$ may be better accounted for by an increase in the constriction degree in the low pharyngeal area in the vicinity of the epiglottis. It is then plausible that the tongue lowering in a Cartesian vowel space may indeed increase the constriction degree in the low pharyngeal area, which would result in backing in the acoustic space (lowered F2). According to the nomogram, based on a two-tube model (e.g., Fant 1960), it is predicted that F2 as a back-cavity resonance for /a/ increases as the length of the back-cavity gets shorter (Stevens, 1989; Ladefoged, 1996; Johnson, 1997). This possibility is indeed further supported by the boundary effects on /a/ in domain-initial positions. Recall that the two speakers $(\mathrm{S} 3, \mathrm{~S} 5)$ who showed accent-induced (articulatory) tongue lowering were the ones who also showed backing in the acoustic space (lowered F2) for /a/, which suggests a close relationship between the articulatory tongue lowering and the backing in the acoustic space, at least in these two speakers.

Turning to /i/, there was a closer mapping between the acoustic and articulatory vowel spaces. The accent-induced fronting effect (enhancement of [-back]) in the acoustic space was further manifested in the tongue fronting in the articulatory $x$ dimension, whereas the nonraising effect (thus no evidence of [+high] enhancement) in F1 was commensurate to the nonraising effect in the articulatory $y$ dimension. In fact, two speakers showed accent-induced lowering effects in both the acoustic (F1) and the articulatory (TM, tongue mid) vowel spaces (and two others in the acoustic space only). The tongue lowering for $/ \mathrm{i} /$ in TM for these speakers might be interpreted as the entire tongue body being shifted forward along the arc of the palate (as evidenced in the tongue fronting), which may rotate the tongue midposition slightly downward. Alternatively, the lowering of TM for accented /i/ could be interpreted, not in terms of place feature enhancement, but simply as a byproduct due to the tongue shifting to achieve a proper constriction degree and location in the area of the palate. However, these two alternatives do not fully explain why there is also a corresponding acoustic lowering effect (F1 raising) in the acoustic dimension. Instead, the acoustic and articulatory lowering effects observed in some speakers may be interpreted as a result of the articulatory maneuver coupled with the jaw lowering (and the lip opening) to increase sonority.

These hypotheses lead to an interesting observation. In the height dimension in both acoustic and articulatory, when a vowel has a place feature whose enhancement is in direct conflict with sonority expansion (i.e., [+high] for /i/), the enhancement of that feature appears to be suppressed, as found in most speakers' data. What appears to be enhanced then is the [-back] feature evident in both the acoustic and articulatory fronting, which at least has no antagonistic effect on sonority expansion. On the other hand, when a vowel has place features whose enhancement is not in conflict with sonority expansion (in the case of $[+\mathrm{low}]$ and $[+$ back $]$ for $/ \mathrm{a} /$ ), no such constraint applies. This observation is also supported by a finding reported by de Jong (1995a), who showed that the tongue position for English vowel / / (with two place features $[+$ high $]$ and $[-$ back $]$ ) is backer but not necessarily higher under accent. ${ }^{3}$

This account suggests that accent-driven hyperarticulation of vowels in English is achieved by an enhancement of some, but not all, place features, with consistent sonority expansion. If the ultimate goal of accent is to maximize phonemic contrast and ultimately lexical distinctions, in the spirit of the hyperarticulation hypothesis, such distinctions are not necessarily maximized by enhancement of all the distinctive place features, but may actually be best enhanced by making segments louder as a result of sonority expansion, and other featural enhancement may enhance the peripherality of the vowels not in all directions but in ways that do not conflict with sonority expansion. Similarly, Fowler (1995) suggests that that the phonetic goal of accent is to simply maximize prominence in order for listeners to understand better (the global effect hypothesis).

\section{B. Prosodic boundary and featural enhancement}

Another hypothesis set forth for this study was that the phonetic realization of vowels at domain edges is driven primarily by sonority expansion, and not by hyperarticulation, such that all three articulators (the tongue, the jaw, and the lips) converge on a larger vocal tract opening at higer prosodic boundaries, resulting in acoustic lowering (i.e., F1 raising). In this vein, it was also predicted that nonsonority (place) features would not be enhanced if the results would be antagonistic to the sonority expansion. However, the results revealed that although there is some evidence for boundary-induced sonority expansion, this is not the only effect seen at the boundaries.

The lip opening was found to be generally larger for both /i/ and /a/ at a higher boundary in both the domain initial and final positions, suggesting that sonority expansion is associated with edges of prosodic domains. Interestingly, however, unlike the accent effect, neither /i/ nor /a/ showed 
any significant increase in the jaw opening at a higher boundary in both the domain initial and final positions. Instead, /i/ showed a significant decrease in jaw opening at a higher boundary.

With respect to the enhancement of place features, /a/ showed lowering and backing effects in both the acoustic and the articulatory vowel spaces (more consistently so in domain-final than domain-initial position), which could be interpreted as the enhancement of $[+$ low $]$ and $[+$ back $]$ as well as sonority expansion. The vowel /i/ also showed acoustic raising and fronting and articulatory raising (but not consistent fronting). This again can be interpreted as the enhancement of $[+$ high $]$ and $[-$ back $]$ at a higher boundary. Thus, from these results we can postulate that it is not only sonority-related features but also nonsonority features that are enhanced at a strong prosodic boundary, even if the enhancement of such nonsonority features (e.g., [+high] for /i/) works antagonistically with sonority expansion. This suggests that sonority is not the only driving force for strengthening effects at prosodic boundaries, rejecting the hypothesis of boundary phenomena as being governed by sonority only (e.g., Farnetani and Vayra, 1996). [The tongue raising for English /i/ is compatible with French EPG data reported in Fougeron (2001), in that the French /i/ in the /p\#_p/ context tends to have a greater degree of linguopalatal constriction at a higher boundary.]

It is also noteworthy that the decreased jaw opening at a higher boundary for /i/ indicates that, if anything, there is an additional adverse effect against boundary-induced sonority expansion for /i/. This is especially interesting because there is a discrepancy between the lip and the jaw opening maxima: /i/ is generally associated with a larger lip opening but a smaller jaw opening. One possibility might have to do with competing articulatory goals in the production of domain-edge /i/: boundary-driven sonority expansion versus the enhancement of the proper constriction degree required for $/ \mathrm{i} /$. It is plausible that the lip opening satisfies boundarydriven sonority expansion while the jaw contributes to an enhancement of [+high] for /i/. (Recall that for the accent effect on /i/, there was neither acoustic nor tongue/jaw raising.) The reduced jaw opening may also have an effect of reinforcing the unroundedness of /i/ that may be responsible at least in part for the increased F2 for preboundary /i/.

Asymmetry in domain-initial versus domain-final prosodic strengthening. As summarized above, the boundary effect is generally more robust domain-finally than domaininitially. In almost all cases, the observed boundary phenomena were evident in more speakers domain-finally than domain-initially. The weaker domain-initial results are roughly consistent with the EPG data in Fougeron and Keating (1997), which showed that the vocal tract opening for /no/ (in reiterant speech), was larger for phrase-initial positions than for phrase-medial positions only for one speaker out of three. This is presumably because the effect of domain-initial strengthening is robust primarily on the domain-initial consonant in $\sharp \mathrm{CV}$, but the degree of strengthening may wane when it comes to the vowel articulation. (See Byrd, 2000; Byrd and Saltzman, 2003 for a possible explanation in the framework of a mass-spring gestural model.) This does not mean to say that there is no domaininitial strengthening effect on the vowel in $\sharp \mathrm{CV}$, but to say that the effect is less robust as compared to the domain-final articulation. [Note that Cho (in press) also showed boundaryinduced strengthening in $\sharp C V$ lip opening kinematics.]

Returning to domain-final phenomena, it has been proposed that domain-final articulation is explained primarily by temporal expansion (e.g., Edwards et al., 1991; Beckman et al., 1992). However, as described above, the results of the present study do indicate that domain-final articulation is marked not only by temporal expansion but also by the enhancement of sonority and place features at a higher prosodic boundary-that is, by strengthening as well as lengthening. A similar domain-final strengthening effect on French /a/ was reported in Tabain (2003). However, the results of the present study are not fully comparable with previous studies. For example, consider the remark of Beckman et al. (1992) regarding domain-final articulation that " $[\mathrm{u}]$ nlike the greater length of a nuclear-accented syllable (with a greater articulatory displacement), intonation-phrase-final lengthening is not accompanied by any significant difference in articulator displacement." The basis for this remark was that the jaw closing gesture for "pop" is temporally longer, but not spatially larger domain-finally. However, this remark does not seem to pertain to our tongue and lip opening data. One possible explanation is that the discrepancy comes from the difference in syllable structure as well as the articulatory parameters. The Beckman et al. conclusion was based on the V-to-C closing displacement data with closed syllables (/pap/) whereas the data in the present study were obtained from the articulatory (static) maxima with open syllables $(/ \mathrm{ba} \# /)$. Another possibility is that the jaw may be less sensitive to changes in the level of prosodic boundary than other articulators. The data presented in this paper show that among the three articulators that were examined, it is only the jaw maxima that do not show expanded articulation for domain-final /a/ (only one out of five speakers showed the jaw lowering effect), showing less sensitivity of the jaw to prosodic boundaries. This is consistent with Tabain's (2003) claim that the jaw is less sensitive to prosodic boundaries than is the tongue. Given these two possible explanations, the findings of the current study need not be seen as contradicting those of Beckman et al., but as complementing them.

\section{Boundary versus accent-induced strengthening}

We have seen that vowels are more extremely articulated when they are either accented or located at a higher prosodic boundary, suggesting that there is some sort of prosodic strengthening. One of the major goals of this paper was to examine whether the prosodic strengthening that occurs at the edges of prosodic domains can be accounted for by the same principle that governs accent-induced strengthening.

The results suggest that the two aspects of prosodic structure are indeed differentiated by distinct phonetic patterns. One of the most comprehensible differences between the two effects comes from the way in which the high front vowel /i/ is produced. Accented /i/ was associated primarily with fronting in both the acoustic and the articulatory vowel spaces, which was accompanied by an increase in both the 
jaw and the lip opening. On the other hand, domain-final /i/ at higher boundaries was associated primarily with raising in both the acoustic and the articulatory spaces, this time accompanied by a decrease in the jaw opening but an increase in the lip opening. We then infer that phonemic distinction is maximized primarily via enhancement of the frontedness ([-back]) under accent but via enhancement of the height $([+$ high $])$ at higher boundaries, while sonority is expanded in both contexts. For /a/, the difference between the accent and the boundary effects was less clear, but the obvious difference was that while there was a clear jaw opening effect for accented /a/, there was virtually no jaw lowering effect for domain-final /a/. Despite all these differences, however, there is one common property that characterizes prosodic strengthening, regardless of whether it comes from accentuation or boundary: vowels are produced with an increase in sonority as evident in the lip opening, which is taken to be a consistent way of increasing linguistic prominence at prosodically strong locations.

\section{Maintenance of phonological contrasts}

Before concluding this paper, it is worth discussing how the results of the present study can be linked to current phonological theories with respect to prosodically strong positions. In the recent phonological literature, prosodically strong positions have been considered by some phonologists as "privileged" or "licensing" positions in which phonological contrasts are most often maintained, in which segments act as triggers of phonological modification such as vowel harmony, and in which they themselves often resist such a modification (e.g., Beckman, 1998; Steriade, 1999; Barnes, 2002). Although it is still controversial whether such a positional privilege is phonetically grounded (being attributable to the richness of the phonetic cues associated with that position) or structurally driven (being purely attributable to the position itself), what is clearer is that, cross-linguistically, phonological contrasts are likely to be maintained in prosodically strong positions. This phonological view of strong positions as licensing for phonological contrasts has been attested in many languages, and is indeed in line with the prosodic strengthening effects in English observed in the present study: the prosodically conditioned enhancement of vowel features can be seen as maximally maintaining phonological contrasts in prosodically strong positions. The propensity of contrast maintenance is also evident in V-to- $\mathrm{V}$ coarticulatory resistance in prosodically strong positions (Cho, 2004). Moreover, such phonological theories may be further enriched by taking into account two aspects of prosodic strengthening found in the present study: prosodic strengthening has different phonetic effects depending on prosodic position and the degree of strengthening at domain edges varies with the level of the prosodic boundary. With respect to the interaction between prosodic strengthening and language-specificity, Cho and McQueen (in press) showed that VOTs tend to be shortened in strong prosodic positions in Dutch, as opposed to those in similar strong positions in English, which suggests that prosodically driven enhancement of phonological contrast (e.g., voiced/voiced phonemic distinction) is further modulated by a language-specific fea- ture system. These findings together suggest that the investigation of cross-linguistic phonological patterns is warranted, with special attention paid to whether positional effects on phonological alternations and contrast maintenance are further conditioned by multiply-layered higher-level prosodic structure.

\section{E. Conclusion}

The results of this study suggest that the complexity of prosodic structure reflected in accentuation and hierarchically nested prosodic constituents appear to be indeed differentiated by speakers, as evident in discrete prosodic strengthening phenomena in different prosodic contexts (in accented syllables versus at edges of prosodic domains; domaininitially versus domain-finally). Building on previous findings, this implies that not only suprasegmental features (duration, pitch, loudness) but also fine-grained segmental phonetic cues are all manipulated to signal the linguistic structure of prosody. Another implication of this study is that prosodic strengthening is not simply a low-level phonetic event but a complex linguistic phenomenon, giving rise to the enhancement of phonological features. This implies that prosodic strengthening is related with linguistic prominence such that it makes a sound more distinct from neighboring segments (syntagmatic enhancement) and/or makes the sound distinct from other contrastive sounds in the language (paradigmatic/phonemic enhancement). This, in turn, means that the phonological theory of positional strength or licensing may need to be further elaborated by taking a high-level prosodic structure into account.

\section{ACKNOWLEDGMENTS}

This work has been supported by the Max Planck Institute for Psycholinguistics, and the NSF doctoral improvement grant (BCS-0001716) to the author and Patricia Keating. The author would like to thank Pat Keating and Sun-Ah Jun for their comments and advice; Adam Albright, Peter Ladefoged, Bruce Hayes, Ken de Jong, and Elizabeth Johnson for valuable suggestions on an earlier version of this paper. This work has been greatly improved by comments and criticisms from Anders Löfqvist, Mary Beckman, and an anonymous reviewer. The articulatory data were originally collected for the author's 2001 UCLA Ph.D. dissertation, but have been completely reprocessed and reanalyzed along with additional acoustic data. Therefore, the results reported jere are different from those in the authors' dissertation.

\footnotetext{
${ }^{1}$ But, See Harrington et al. (2000) in which one speaker showed temporal sequencing in achieving the sonority expansion first and then the hyperaticulation of the vowel features of accented /i/ in Australian English.

${ }^{2}$ Out of 320 sentences that contained a phrase boundary IP or ip), 302 sentences $(94.3 \%)$ reached agreement. Each speaker contributed 28 to 32 IP sentences and 29 to 31 ip sentences, showing a balanced distribution across boundary types. Furthermore, sentences were also distributed roughly equally across accent conditions: ACC\#ACC /a/, 36 sentences; /i/, 39 sentences), UNA\#ACC /a/, 38 sentences; /i/, 38 sentences), UNA\#ACC /a/, 36 sentences; /i/, 39 sentences) and UNA\#UNA /a/, 38 sentences; /i/, 38 sentences).

${ }^{3} 3$ This parallel should be taken with caution: The tongue height dimension in de Jong (1995a) was defined by a line of pellets on the lower molars
} 
whereas the tongue height dimension in the present was defined by the occlusal plane.

Barnes, J. (2002). "Positional neutralization: A phonologization approach to typological patterns," Ph.D. dissertation, University of California, Berkeley.

Beckman, J. N. (1998). "Positional faithfulness," Ph.D. dissertation, University of Massachusetts, Amherst.

Beckman, M. E. (1996). "The parsing of prosody," Lang. Cognit. Processes 11, 17-67.

Beckman, M. E., and Ayers, G. (1997). "Guidelines for ToBI labeling, version 3.0," unpublished Manuscript, Ohio State University.

Beckman, M. E., and Edwards, J. (1994). "Articulatory evidence for differentiating stress categories," in Papers in Laboratory Phonology III: Phonological Structure and Phonetic Form, edited by P. A. Keating (Cambridge University Press, Cambridge), pp. 7-33.

Beckman, M. E., Edwards, J., and Fletcher, J. (1992). "Prosodic structure and tempo in a sonority model of articulatory dynamics," in Papers in Laboratory Phonology II: Gesture, Segment, Prosody, edited by G. Docherty and D. R. Ladd (Cambridge University Press, Cambridge), pp. 68-86.

Beckman, M. E., and Pierrehumbert, J. (1986). "Intonational structure in Japanese and English," Phonol. Yearbook 3, 255-309.

Bolinger, D. (1970). "Relative height," in Prosodic Feature Analysis. Montreal, edited by P. Léon, G. Faure, and A. Rigault (Marcel Didier), pp. 109-127 (reprinted in Bolinger (1972). Intonation: Selected Readings (Penguin Books, Harmondsworth).

Browman, C. P., and Goldstein, L. (1992). "Articulatory phonology: an overview," Phonetica 49, 155-180.

Byrd, D. (2000). "Articulatory vowel lengthening and coordination at phrasal junctures," Phonetica 57, 3-16.

Byrd, D., and Saltzman, E. (2003). "The elastic phrase: Modeling the dynamics of boundary-adjacent lengthening," J. Phonetics 31, 149-180.

Cho, T. (2002). The Effects of Prosody on Articulation in English (Routledge, New York).

Cho, T. (in press). "Manifestation of prosodic structure in articulation: Evidence from lip kinematics," in Laboratory Phonology 8: Varieties of Phonological Competence, edited by L. M. Goldstein, D. H. Whalen, and C. T. Best (Mouton de Gruyter, Berlin/New York).

Cho, T. (2004). "Prosodically conditioned strengthening and vowel-tovowel coarticulation in English," J. Phonetics 32, 141-176.

Cho, T., and Jun, J. (2000). "Domain-initial strengthening as featural enhancement: Aerodynamic evidence from Korean," in Chicago Linguistics Society, Vol. 36, No. 1, pp. 31-44.

Cho, T., and Keating, P. (2001). "Articulatory and acoustic studies of domain-initial strengthening in Korean,” J. Phonetics 29, 155-190.

Cho, T., and McQueen, J. M. (in press). "Prosodic influences on consonant production in Dutch: Effects of prosodic boundaries, phrasal accent and lexical stress," J. Phonetics 33.

Chomsky, N., and Halle, M. (1968). The Sound Pattern of English (Harper \& Row, New York).

de Jong, K. (1995a). "The supraglottal articulation of prominence in English: Linguistic stress as localized hyperarticulation," J. Acoust. Soc. Am. 97, 491-504.

de Jong, K. (1995b). "On the status of redundant features: the case of backing and rounding in American English," in Phonology and Phonetic Evidence: Papers in Laboratory Phonology IV, edited by B. Connell and A. Arvaniti (Cambridge University Press, Cambridge, UK), pp. 68-86.

Edwards, J. E., Beckman, M. E., and Fletcher, J. (1991). "The articulatory kinematics of final lengthening," J. Acoust. Soc. Am. 89, 369-382.

Edwards, J., and Beckman, M. E. (1988). "Articulatory timing and the prosodic interpretation of syllable duration," Phonetica 45, 156-174.

Erickson, D. (2002). "Articulation of extreme formant patterns for emphasized vowels," Phonetica 59, 134-149.

Fant, G. (1960). The Acoustic Theory of Speech Production (Mouton, The Hague).

Farnetani, E., and Vayra, M. (1996). "The role of prosody in the shaping of articulation in Italian CV syllables," Proceedings of the 1st ESCA Workshop on Speech Production Modeling, 4th Speech Production Seminar, Autrans, France, pp. 9-12.

Fougeron, C. (2001). "Articulatory properties of initial segments in several prosodic constituents in French," J. Phonetics 29, 109-135.
Fougeron, C., and Keating, P. A. (1997). "Articulatory strengthening at edges of prosodic domains," J. Acoust. Soc. Am. 101, 3728-3740.

Fowler, C. A. (1995). "Acoustic and kinematic correlates of contrastive stress accent in spoken English," in Producing Speech: Contemporary Issues: For Katherine Safford Harris, edited by F. Bell-Berti and J. J. Raphael, pp. 355-373.

Harrington, J., Fletcher, J., and Beckman, M. E. (2000). "Manner and place conflicts in the articulation of accent in Australian English," in Acquisition and the Lexicon: Papers in Laboratory Phonology V, edited by M. B. Broe and J. B. Pierrehumbert (Cambridge University Press, Cambridge), pp. $70-87$.

Hoole, P. (1996). "Issues in the acquisition, processing, reduction and parameterization of articulographic data," Forschungsberichte des Instituts für Phonetik und Sprachliche Kommunikation, München, Vol. 34, pp. $158-173$.

Jackson, M. T. T. (1988). "Analysis of tongue positions: Language-specific and cross-linguistic model," J. Acoust. Soc. Am. 84, 124-143.

Johnson, K. (1997). Acoustic and Auditory Phonetics (Blackwell, Oxford).

Jun, S.-A. (1993). "The phonetics and phonology of Korean prosody," Ph.D. dissertation, Ohio State University.

Jun, S.-A. (1998). "The accentual phrase in the Korean prosodic hierarchy," Phonology 15, 189-226.

Keating, P. A., Cho, T., Fougeron, C., and Hsu, C. (1999). "Domain-initial strengthening in four languages," UCLA Working Papers in Phonetics, Vol. 97, pp. 139-151 (also to appear in Papers in Laboratory Phonology 6, Cambridge (Cambridge University Press, Cambridge).

Ladefoged, P. (2000). A Course in Phonetics, 4th ed. (Harcourt, Brace, Jovanovich, Fort Worth, TX).

Ladefoged, P. (1996). Elements of Acoustic Phonetics, 2nd ed. (The University of Chicago Press, Chicago).

Löfqvist, A. (1999). "Interarticulator phasing, locus equations, and degree of coarticulation," J. Acoust. Soc. Am. 106, 2022-2030.

Löfqvist, A., Gracco, V. L., and Nye, P. W. (1993). "Recording speech movements using magnetometry: One laboratory's experience," Forschungberichte des Instituts für Phonetik und Sprachliche Kommunikation der Universität München, Vol. 31, pp. 143-162.

Neary, T. N. (1977). Phonetic Feature Systems for Vowels, Ph.D. dissertation. University of Connecticut, Storrs, CT.

Pierrehumbert, J. (1980). "The phonology and phonetics of English intonation," Ph.D. dissertation, MIT.

Pierrehumbert, J., and Beckman, M. E. (1988). Japanese Tone Structure (MIT Press, Cambridge, MA).

Selkirk, E. (1986). "On derived domains in sentence phonology," Phonol. Yearbook 3, 371-405.

Shattuck-Hufnagel, S., and Turk, A. E. (1996). "A prosody tutorial for investigators of auditory sentence processing," J. Psycholinguist. Res. 25, $193-247$

Silverman, K., Beckman, M. E., Pitrelli, J., Ostendorf, M., Wightman, C., Price, P., Pierrehumbert, J., and Hirschberg, J. (1992). "TOBI: A standard for labeling English prosody," in Proceedings of the 1992 International Conference on Spoken Language Processing, Vol. 2, pp. 867-870.

Steriade, D. (1999). "Phonetics in phonology: The case of laryngeal neutralization," in UCLA Working Papers in Linguistics, No. 2, Papers in Phonology 3, edited by M. Gordon, pp. 25-146.

Stevens, K. N. (1989). "On the quantal nature of speech," J. Phonetics 17, $3-45$.

Tabain, M. (2003). "Effects of prosodic boundary on /aC/ sequences: articulatory results," J. Acoust. Soc. Am. 113, 2834-2849.

Turk, A. E., and Shattuck-Hufnagel, S. (2000). "Word-boundary-related durational patterns in English," J. Phonetics 28, 397-440.

Westbury, J. R. (1994). "On coordinate systems and the representation of articulatory movements,” J. Acoust. Soc. Am. 95, 2271-2273.

Wightman, C. W., Shattuck-Hufnagel, S., Ostendorf, M., and Price, P. J. (1992). "Segmental durations in the vicinity of prosodic phrase boundaries," J. Acoust. Soc. Am. 91, 1707-1717.

Wood, S. (1979). "A radiographic analysis of constriction locations for vowels," J. Phonetics 7, 25-43.

Wood, S. (1982). "X-ray and model studies of vowel articulation," Ph.D. dissertation, Lund University (see also Working Papers, Vol. 23, pp. 1-192, Dept. of Linguistics and Phonetics, Lund University). 